\title{
Adult Necrotising Enterocolitis in a Breast Cancer Patient after First Cycle of Adjuvant Chemotherapy
}

\author{
Fatima Tuz Zahra Shakir, Rizwan Sultan, Riaz Siddiqui, M. Zubair Shah and Ayesha Javed \\ Department of Surgery, Islamabad Medical Complex, Nescom, Islamabad, Pakistan
}

\begin{abstract}
Chemotherapy-induced diarrhea has become a worrisome problem for the patients as well as the treating surgeons and oncologists because patient outcome is significantly affected. Necrotising enterocolitis is a rare disease in adults. It is now termed as neutropenic enterocolitis in adults, because it is seen more often as a complication of aggressive systemic chemotherapy. It is believed to be associated with chemotherapy-induced intestinal mucosal injury followed by a super infection, which leads to bacteremia, and majority of these patient develop neutropenia. They often have vague and nonspecific presentation which gets overshadowed by the symptoms of primary malignant disease and expected toxic side effects of chemotherapy. In this case report, we discuss the case of a middle-aged female with infiltrating ductal carcinoma of breast, who on adjuvant chemotherapy, developed necrotising enterocolitis requiring emergency exploratory laparotomy. The objective of this study is to increase awareness regarding this fatal complication of chemotherapy because very little research has been done so far. We also review literature to find out the cause of necrotising enterocolitis in reported cases. It is recommended to treat diarrhea, caused by chemotherapy, with antibiotics instead of just loperamide because of decreased immunity of the patient, which could lead to fatal complications.
\end{abstract}

Key Words: Chemotherapy, Diarrhea, Necrotising enterocolitis, Adults, Cancer.

How to cite this article: Shakir FTZ, Sultan R, Siddiqui R, Shah MZ, Javed A. Adult necrotising enterocolitis in a breast cancer patient after first cycle of adjuvant chemotherapy. J Coll Physicians Surg Pak 2019; 29(10):1006-8.

\section{INTRODUCTION}

Chemotherapy is a necessary evil in the treatment of cancer and its role is increasing with the passage of time due to discovery of new and aggressive medicines to eradicate cancer cells. These drugs cause significant adverse effect on other organs of the body. Chemotherapy-induced diarrhea occurs in $40-50 \%$ of patients depending upon the regimen given. It can cause depletion of fluids and electrolytes leading to malnutrition, dehydration, and hospitalisation. In addition, diarrhea can interfere with cancer treatment by causing delay or reduction of dose, which has an impact on survival. Therapeutic or prophylactic measures, according to current guidelines, include supportive treatment with loperamide and octreotide. ${ }^{1}$ Loperamide is an opioid which functions by decreasing intestinal motility by directly affecting the smooth muscle of the intestine. Octreotide is a synthetic somatostatin analog which decreases secretion of a number of hormones, such as vasoactive intestinal peptide (VIP); prolongs intestinal transit time and reduces secretion and increases absorption of fluid and electrolytes.

Correspondence to: Dr. Fatima Tuz Zahra Shakir, Department of Surgery, Islamabad Medical Complex, Nescom, Islamabad, Pakistan

E-mail: fatimatuzahra04@gmail.com

Received: November 28, 2018; Revised: March 11, 2019; Accepted: March 28, 2019
Neutropenia, induced by chemotherapy, is also one of the common complications encountered after chemotherapy. Neutropenia due to malignant disease and low immunity exposes the cancer patient to opportunistic infections. Necrotising enterocolitis is described as transmural inflammation of the large intestine in patients with low neutrophil count who are suffering from severe myelosuppression.2 Almost whole gut can be compromised, but most commonly involved areas are from terminal ileum to ascending colon. ${ }^{3}$ Necrotising enterocolitis usually occurs in the $1^{\text {st }}$ to $2^{\text {nd }}$ week after chemotherapy due to low neutrophil count. The exact etiology and development of the disease is not known but profound neutropenia and growth of opportunistic organisms appear to be the most dominant reason. ${ }^{4}$

We herein, present a case of a 35-year-old female with infiltrating ductal carcinoma who developed necrotising enterocolitis following first cycle of adjuvant chemotherapy.

\section{CASE REPORT}

A 35-year-old married and nulliparous female developed a firm to hard $3 \times 4 \mathrm{~cm}$ large lump in her right breast. After workup, she got diagnosed with infiltrating ductal carcinoma of breast with no metastasis; and had modified radical mastectomy with level two axillary clearance. Two months later, she received first cycle of chemotherapy, which was injection doxetol, zycram, and adriablastina. All of these drugs cause diarrhea among 
many other side effects, for which she was prescribed imodium ${ }^{\circledR}$ for post-chemotherapy diarrhea on discharge. Eleven days later, she developed severe generalised abdominal pain and distention associated with constipation. She underwent conservative treatment from general physician of local hospital and her pain settled. However, two days later, her symptoms reappeared, much worse than before and she came to tertiary care hospital for management. On examination, she was tachypneic, tachycardic and hypotensive. She had generalised abdominal tenderness, rigidity and absent bowel sounds. On workup, there were normal white cells and neutrophilic count with normal renal functions and serum electrolytes. X-ray abdomen showed massive air under right diaphragm, which mandated urgent surgical intervention (Figure 1). Patient underwent emergency exploratory laparotomy, which revealed dusky gangrenous perforated caecum, fecal peritonitis, pus, sloughing off of serious coat of intestines and fibrin exudates; with primary arterial supply of gut uncompromised (Figure 2). Right hemi-colectomy was done. Terminal ileum and transverse colon were brought out as double barrel stoma. Postoperative period was prolonged which consisted of conservative management with intravenous painkillers and fluids. Intravenous antibiotics like colistin sulphate and amikacin were administered according to

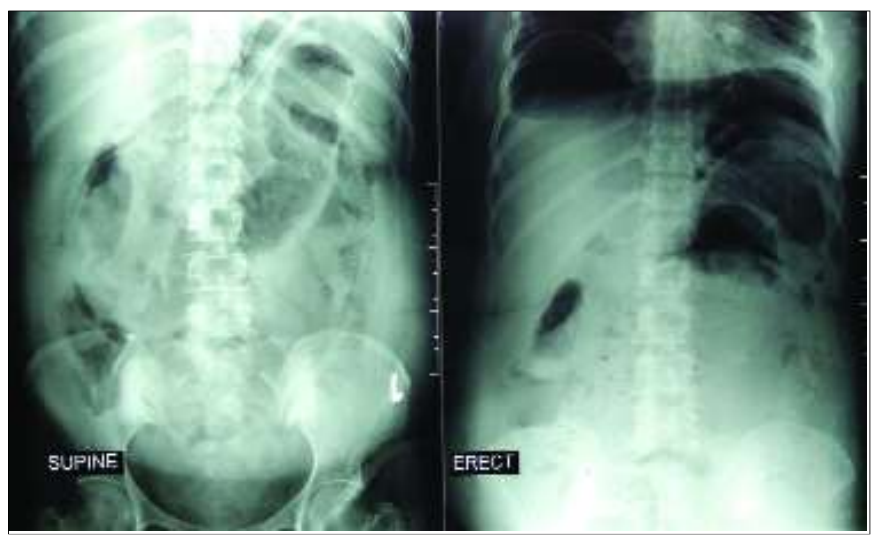

Figure 1: X-ray abdomen showing gas under right hemidiaphragm indicating gut perforation.

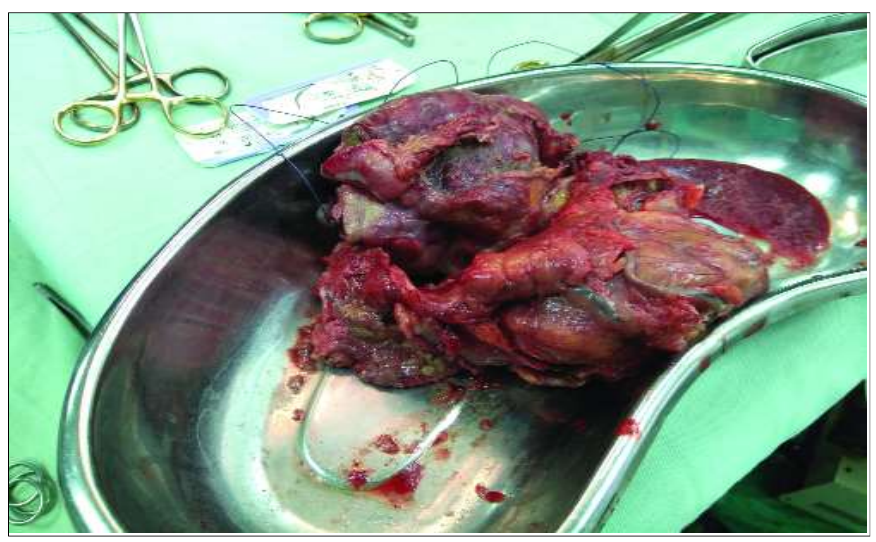

Figure 2: Dusky perforated caecum with fibrin exudates. pus culture and sensitivity report, which was positive for Klebsiella and showed resistance to majority of other antiobiotics. Histopathology revealed acute on chronic non-specific inflammation and perforation of caecum, ulceration with fibrinoid necrosis, granulation tissue and congestion. There was no evidence of malignancy or metastasis from primary disease. After three months, stoma reversal was performed along with side to side anastomosis between terminal ileum and transverse colon. Bowel continuity and function was eventually restored. Patient is clinically and symptomatically better. She will receive her remaining 5 cycles of chemotherapy as part of cancer treatment on follow-up.

\section{DISCUSSION}

The exact etiology of necrotizing enterocolitis is unknown, but it is widely hypothesized that it is associated with chemotherapy-induced intestinal mucosal injury, followed by a superinfection, which eventually leads to sepsis in cancer patients. Bacteria are mostly responsible but fungal and viral pathogens are also implicated and isolated in many cases. The common organisms involved are bacteria like Klebsiella, Escherichia coli, Enterobacter, Pseudomonas, Clostridia and Staphylococcus epidermidis and viruses like Corona and Rota viruses. 5 Cancer patients who receive chemotherapy have weakened defence system. There is increased bacterial load due to overgrowth of gut bacteria. This overgrowth results in diarrhea, which is treated with loperamide, a gut motility slowing agent resulting in increased infective material in the gut, followed by severe inflammation of gastrointestinal tract and perforation with sepsis. Caecal perforation is a rare cause of peritonitis but in case of severe immunocompromised state, it is possible as reported above. Early diagnosis can be aided by ultrasound and CT scan which show bowel wall thickening. Bowel wall thickness of more than $4 \mathrm{~mm}$ and intestinal pneumatosis are indicative of necrotising enterocolitis. ${ }^{6}$ It is almost impossible to diagnose the disease at an early stage as these patients initially ignore signs and symptoms of gastrointestinal upset and only present late with lifethreatening complications which require urgent surgical intervention. Only peroperative findings or autopsies confirm the diagnosis of necrotising enterocolitis. ${ }^{7}$

The cases of necrotising enterocolitis are commonly reported at 7 th day post-chemotherapy. The mortality is reported to be as high as $45 \%$ in case studies. Risk factors include drug related mucosal injury, cytopenias, disseminated intravascular coagulation (DIC), and steroid therapy. Necrotising enterocolitis is now reported in treated cases of metastatic breast cancer, non-smallcell lung carcinoma, ovarian, esophageal and incurable stage of prostate cancer, while on chemotherapy. It is now seen with the use of many chemotherapy agents like taxenes, pegylated interferon, cytosine arabinoside, 
vinca alkaloids, sulphasalazine, procainamide, gemcitabine, pemetrexed, 5-fluoro uracil, epirubicin, cyclophosphamide, platinum derivatives, vinorelbine, ifosfamide, etoposide and alemtuzumab.2,4,8,9 It has been described most recently in patients with solid tumors who receive taxenes alone or in combination.

Literature review till date shows about 72 reported cases of adult necrotising enterocolitis; out of these, 12 cases were seen in cancer patients during the course of chemotherapy and two cases of necrotising enterocolitis specifically reported gastrointestinal perforation after loperamide therapy. ${ }^{10}$ Hence, the role of loperamide in the pathogenesis of complications is suggested. We recommend treatment of chemotherapy-induced diarrhea with antibiotic instead of loperamide to prevent vicious cycle of necrotising enterocolitis. The use of antidiarrheal agents and narcotic analgesic masks or confounds the clinical picture, eventually leading to death. New strategies and effective agents are under development to reduce the morbidity and mortality associated with chemotherapy-induced diarrhea. Further research focusing on the prophylactic use of antibiotics, budesonide, probiotics or activated charcoal is absolutely necessary to define the role of these drugs in routine clinical setting for management of this debilitating toxicity.

Adult necrotising enterocolitis, although a rare disease, seems to have a relatively high incidence in cancer patients receiving chemotherapy. More research needs to be done to understand the disease process and develop appropriate guidelines for management. Awareness regarding this disease among treating physicians in high risk patients is essential, because only then it can lead to early diagnosis and prompt treatment. Chemotherapy-induced diarrhea should be treated with antibiotics and proper patient counselling and regular follow-up is necessary to prevent lifethreatening complications.

\section{PATIENT'S CONSENT:}

Informed consent was taken from the patient about this case report.

\section{CONFLICT OF INTEREST:}

Authors declared no conflict of interest.

\section{AUTHORS' CONTRIBUTION:}

FTZS, RS: Substantially contributed to the conception or design of the work; acquisition, analysis, or interpretation of data for the work; drafted the work or revised it critically for important intellectual content; finally approved the version to be published; agreed to be accountable for all aspects of the work in ensuring that questions related to the accuracy or integrity of any part of the work are appropriately investigated and resolved.

RS, MZS, AJ: Finally approved the version to be published; agreed to be accountable for all aspects of the work in ensuring that questions related to the accuracy or integrity of any part of the work are appropriately investigated and resolved.

\section{REFERENCES}

1. Alexander S. Chemotherapy-induced diarrhea: Pathophysiology, frequency and guideline-based management. Ther Adv Med Oncol 2010; 2:51-63.

2. Mehdi I, Al Bahrani B. Chemotherapy-induced neutropenic necrotizing enterocolitis: A review. J Pak Med Assoc 2012; 62: 718-23.

3. Alt B, Glass NR, Sollinger H. Neutropenic enterocolitis in adults: Review of the literature and assessment of surgical intervention. Am J Surg 1985; 149:405-8.

4. Rodrigues FG, Dasilva G, Wexner SD. Neutropenic enterocolitis. World J Gastroenterol 2017; 23:42-7.

5. Zachariah SK. Adult necrotizing enterocolitis and non occlusive mesenteric ischemia. J Emerg Trauma Shock 2011; 4:430-2.

6. Gorschluter M, Mey U, Strehl J, Ziske C, Schepke M, SchmidtWolf IGH, et al. Neutropenic enterocolitis in adults: Systematic analysis of evidence quality. Eur J Haematol 2005; 75:1-13.

7. Cunningham SC, Fakhry K, Bass BL, Napolitano LM. Neutropenic enterocolitis in adults: Case series and review of the literature. Dig Dis Sci 2005; 50:215-20.

8. Maeta M, Mizusawa K, Koga S. Induction of diffuse necrotizing enterocolitis by anticancer chemotherapy. Gastroenterol Jpn 1987; 22:370-3.

9. Geisler JP, Schraith DF, Manahan KJ, Sorosky JI. Gemcitabine associated vasculitis leading to necrotizing enterocolitis and death in women undergoing primary treatment for epithelial ovarian/peritoneal cancer. Gynecol Oncol 2004; 92:705-7.

10. Olm M, Gonzalez FJ, Garcia-Valdecasas JC, Fuster J, Bertran A, Milla J. Necrotising colitis with perforation in diarrhoic patients treated with loperamide. Eur J Clin Pharmacol 1991; 40:415-6. 